\title{
Intravenous Push
}

National Cancer Institute

\section{Source}

National Cancer Institute. Intravenous Push. NCI Thesaurus. Code C64995.

A method of drug delivery in which a usually small volume of drug is administered rapidly into a vein. 